\title{
El comportamiento de queja del consumidor mexicano
}

\section{Mexican Consumer Complaint Behavior}

\author{
Ignacio Javier Cruz Rodríguez* (D) https://orcid.org/0000-0003-4889-5838 \\ Eduardo Rosas Rojas ${ }^{* \star}$ (D) https://orcid.org/0000-0002-7255-7778
}

\begin{abstract}
Resumen
Objetivo: examinar los principales determinantes del comportamiento de queja del consumidor en México entre 2006 y 2017 , utilizando datos anuales de las 32 entidades federativas. Metodología: se estimó un modelo de datos de panel balanceado estático y después se incluyó el efecto dinámico que impera en el comportamiento de queja de los consumidores mexicanos mediante el método generalizado de momentos. Resultados: se comprobó que la experiencia previa en las reclamaciones de los consumidores, como resultado de la práctica, les permite aprender y mejorar sus métodos de queja; además, se identifica que los montos menores de recuperación incrementan las reclamaciones. También se registró que los quejosos poseen mayores ingresos que los no quejosos y que son las mujeres con preparación académica quienes más presentan quejas. Limitaciones: la información que se analizó corresponde sólo a los servicios en general y no incluye las quejas sobre los servicios financieros. Valor: la implementación de un modelo de datos de panel dinámico permite superar el problema de endogeneidad entre las variables, lo que da oportunidad de estimar el impacto de las experiencias de quejas previas en las inconformidades actuales. Conclusiones: los resultados obtenidos sobre el comportamiento de queja del consumidor mexicano coinciden con las hipótesis de las principales investigaciones para otros países.

Palabras clave: comportamiento de queja; consumidor; modelos de datos de panel; protección al consumidor.
\end{abstract}

\begin{abstract}
Objective: to examine the main determinants of consumer complaining behavior in Mexico from 2006 to 20I7, using annual data for the 32 states. Methodology: a static balanced panel data model was estimated and later the dynamic effect that prevails in the complaining behavior of Mexicans was included by means of the generalized method of moments. Results: it was proven that consumers' previous complaint experience, as a result of its practice, lets them learn and improve their complaining methods. It is also identified that lower refunded amounts increase the amount of complaints. It was registered that complainers have higher salaries than those who do not complaint and that women with formal education are the ones who complain the most. Limitations: the analyzed information corresponds only to the services in general and the financial services were excluded. Value: the implementation of a dynamic panel data model allows overcoming the endogeneity problem between the variables; this gives the opportunity to estimate the impact of previous complaints experiences on current nonconformities. Conclusions: the results obtained about the Mexican consumer complaining behavior coincide with the hypotheses of the main investigations for other countries.
\end{abstract}

Keywords: complaining behavior; consumer; panel data models; consumer protection.

Cómo citar: Cruz Rodríguez, I. J., y Rosas Rojas, E. (202I). El comportamiento de queja del consumidor mexicano. región y sociedad, 33, el 44I. doi: I0.22198/rys202I/33/I44I

*Universidad Autónoma de Coahuila, Centro de Investigaciones Socioeconómicas. Edificio S. Unidad Camporredondo. Prol. David Berlanga y 16 de Septiembre s. n., Saltillo, Coahuila, México. Correo electrónico: rodriguez.ignacio@uadec.edu.mx

** Autor para correspondencia. Universidad Autónoma del Estado de México. Centro Universitario UAEM Valle de México. Boulevard Universitario s. n., Valle Escondido, Atizapán de Zaragoza, Estado de México, México. Correo electrónico: erosasr@uaemex.mx

Recibido: 26 de enero de 2021

Aceptado: 20 de mayo de 2021

Liberado: 1 de julio de 2021 


\section{Introducción}

En el presente artículo, el comportamiento de queja del consumidor (CQC) se define como la comunicación o las acciones de cualquier otra índole que éste lleva a cabo para mostrar su insatisfacción sobre algún producto o servicio de una empresa u otra entidad (Abney, Pelletier, Ford y Horky, 2017). De acuerdo con Larivet y Brouard (2010), el hecho de quejarse lleva implícito el objetivo de obtener algo a cambio, ya sea una disculpa, un reembolso o algún tipo de descuento.

El CQC puede tener diversos matices. Una de las clasificaciones empleadas para estudiarlo es la propuesta de Singh (1988), quien divide el comportamiento de queja en tres categorías: respuesta de voz, respuesta personal y respuesta ante terceras entidades. La respuesta de voz significa que el quejoso transmite su queja de manera directa a la empresa, al servicio al cliente o se mantiene en silencio y no lleva a cabo acción alguna. En las respuestas privadas los individuos expresan la queja de forma oral a las personas cercanas, a los medios sociales, a las organizaciones o participan en boicots. La respuesta a la tercera entidad implica canalizar la queja a través de leyes o normas usando las instituciones que, en general, velan por los derechos de los consumidores, como las organizaciones de consumidores independientes o las agencias gubernamentales de protección a los derechos del consumidor. En esta investigación se tomarán en consideración las quejas realizadas ante la tercera entidad, debido a que hay estadísticas disponibles cuyo acceso es público.

Para abordar el estado del arte conceptual y metodológico, este estudio analiza las principales investigaciones con el objeto de identificar los determinantes más relevantes del CQC que inducen al consumidor a presentar una inconformidad formal ante la Procuraduría Federal del Consumidor (PROFECO). Se emplearon datos anuales de entre 2006 y 2017 para las 32 entidades federativas de México y se estimaron las pruebas de hipótesis correspondientes mediante la metodología de datos de panel estático y dinámico.

El artículo está estructurado en seis apartados. Además de la presente introducción, en el segundo se expone una revisión de la literatura sobre la expectativa disconforme del CQC y de las investigaciones que abordan el tema de interés. En el tercero se aborda la metodología econométrica de datos de panel estático y dinámico. En el cuarto se muestra el análisis empírico y se reportan los hechos estilizados de la estadística disponible. En el quinto se describen los resultados de los modelos propuestos y se comprueban las hipótesis planteadas. $\mathrm{Y}$ en el sexto se exponen las conclusiones.

\section{Revisión de la literatura}

La investigación pretende comprender la experiencia de insatisfacción de los consumidores mexicanos y cómo un episodio previo de reclamación les dota de conocimientos y mejores prácticas al momento de ejercer una queja ante la PROFECO. En términos teóricos, la hipótesis de la expectativa disconforme 
del comportamiento de queja del consumidor (EDCQC) describe un proceso individual que inicia con la expectativa antes del uso de un producto y termina con la percepción después de su uso. Esta visión teórica afirma que la expectativa antes de la compra determina la insatisfacción. Es decir, si el producto no se ajusta a la expectativa del consumidor, éste estará insatisfecho y podría quejarse (Ashraf, Waseem, Rizwan, Ahmed y Nazeer, 2013; Johnston, 1995; Zeelenberg y Pieters, 2004).

Uno de los primeros documentos que reportaron que las quejas presentadas con anterioridad tienen un impacto en la conducta de los consumidores, fue el de Martin (1991). De la misma manera, Singh y Wilkers (1996) afirman que la experiencia previa de queja actúa de manera cognitiva y predispone al consumidor a quejarse otra vez si vuelve a experimentar insatisfacción con un proceso de compra, ya sea con el mismo proveedor o con cualquier otro.

Jin (2010) argumenta que si un consumidor se queja con frecuencia, va adquiriendo conciencia de su actitud ante la reclamación y también va comprendiendo mejor el proceso. Ursic (1985) sugiere que un consumidor que se queja de manera constante desarrolla una actitud positiva hacia la queja y tendrá más valor para seguir inconformándose y más probabilidades de éxito en comparación con los consumidores que no se quejan con frecuencia. Berry, Seiders y Grewal (2002) afirman que el CQC depende de las experiencias previas de episodios de queja, en particular en las tiendas de servicios.

La visión teórica de Singh y Wilkers (1996) sobre el EDCQC muestra que una vez que los consumidores se han quejado, aprenden a protestar, pues entienden mejor la manera en que funcionan las normas al respecto. Además, si la queja obtuvo beneficios, los consumidores estarán muy dispuestos a volverse a quejar, por lo que se puede esperar que las reclamaciones anteriores tengan un efecto positivo en el comportamiento corriente de insatisfacción. Zeelenberg y Pieters (2004) comentan que si mediante la reclamación el consumidor obtiene un ingreso, éste puede comunicar por diversas vías su experiencia, lo cual podría incentivar a otras personas, ya sean consumidores que nunca se han quejado o consumidores que ya lo han hecho, a externar su insatisfacción.

La literatura sobre los factores que determina el CQC coincide en la importancia que tiene conocer el verdadero mecanismo, sus condicionantes y el nivel de insatisfacción. De acuerdo con Moliner, Gil Berenger y Fuentes (2008), las variables propuestas son múltiples y de diversa índole. Algunas se asocian con los entornos de compra y de consumo, con los elementos culturales, con los aspectos demográficos y sociales, con la durabilidad o con las características de la empresa. Sin embargo, las principales investigaciones (Berry, Seiders y Grewal, 2002; Johnston, 1995 Martin, 1991; Singh y Wilkes, 1996; Zeelenberg y Pieters, 2004) señalan que son las variables relativas al individuo las que en verdad influyen en el comportamiento de inconformidad. Sobresalen el nivel de información y de experiencia, la estimación de la probabilidad de éxito de la queja y la experiencia de quejas anteriores. En su libro Las desventajas de los consumidores, Andreasen (1975) señala: 
La hipótesis del consumidor desfavorecido argumenta que los problemas de los consumidores desfavorecidos son principalmente atribuibles a sus características personales, el tipo de personas que son. Sostiene que el verdadero problema es que los consumidores desfavorecidos son demasiado viejos, pobres, poco educados, poco sofisticados, definitivamente por la raza, etc., para poder ser consumidores efectivos en el mercado urbano. (p. 5)

Las investigaciones de Volkov, Harker y Harker (2005), Garret y Taumanoff (2010) y Kincl y Štrach (2018) se concentran en las variables demográficas de ingreso, nivel de educación y género como los determinantes más relevantes del CQC ante una agencia gubernamental.

La investigación de Cruz y Galán (2019), para el caso de México, confirma que los determinantes del CQC dependen del nivel de agregación de las quejas presentadas. En ese sentido, los autores estiman dos modelos diferentes: el primero a escala de entidad federativa, y el segundo a escala de empresa. Las variables determinantes del CQC a escala estatal son socioeconómicas: el ingreso y el porcentaje de población urbana son las de mayor impacto. A continuación, se describen las que inciden en el CQC y que se han podido recopilar para la muestra que se investiga.

a) Experiencia previa de queja

De acuerdo con Ursic (1985), Martin (1991) y Singh y Wilkes (1996), la experiencia de queja puede tomarse como la probabilidad de que una persona vuelva a inconformarse ante el mismo proveedor de servicios por un nuevo caso de insatisfacción. La experiencia puede afectar la actitud y el comportamiento del consumidor en caso de insatisfacción en el presente o en el futuro. Para Singh y Wilkes (1996) y Berry, Seiders y Grewal (2002), los clientes desarrollan una actitud positiva hacia la queja cuando aprenden cómo interponerla y cuáles son sus beneficios. A medida que el cliente presenta con mayor frecuencia las inconformidades a las agencias gubernamentales, aprende más métodos, su actitud hacia las quejas se fortalece y gana seguridad debido a los conocimientos adquiridos a través de las experiencias, lo que aumenta su expectativa de obtener una reparación por parte de la empresa.

b) Probabilidad de éxito

El trabajo de Hirschman (1970) fue el primero en subrayar que los consumidores valoran los costos y los beneficios de quejarse. Entre las ventajas mencionadas, se encuentra la probabilidad de éxito de la inconformidad, en la que el premio consiste en la materialización de algún beneficio, ya sea el reembolso, el descuento o la reposición del producto o del servicio por el que se quejaron. De acuerdo con Singh y Wilkes (1996), el ingreso que obtengan los quejosos puede representar un incentivo, tanto para los consumidores que se han quejado anteriormente, como para los consumidores que nunca lo han hecho. Esta situación puede afectar el juicio que los consumidores tengan sobre la probabilidad de éxito cuando presentan una queja. De esta manera, la variable se define 
como la probabilidad de obtener una reparación, ya sea el reembolso, un intercambio o una disculpa cuando una queja se registra en una empresa o en una agencia gubernamental (Singh, 1990). Morel, Poiesz y Wilke (1997), Stephens y Gwinner (1998), Maxham III y Netemeyer (2002) y Cruz y Galán (2019) encuentran evidencia estadística de este efecto (véase tabla 1).

Tabla 1. Estudios sobre la probabilidad de éxito de las reclamaciones

\begin{tabular}{|c|c|c|c|}
\hline Autores & $\begin{array}{l}\text { Determinantes } \\
\text { del estudio }\end{array}$ & $\begin{array}{c}\text { Metodología/ } \\
\text { Muestra }\end{array}$ & Resultados \\
\hline $\begin{array}{l}\text { Morel, Poiesz } \\
\text { y Wilke (1997) }\end{array}$ & $\begin{array}{l}\text { Variable dependiente: } \\
\text { Probabilidad de acción } \\
\text { de queja. } \\
\text { Variables independientes: } \\
\text { Importancia de la situación, } \\
\text { nivel de información, } \\
\text { experiencia y probabilidad } \\
\text { de éxito de la reclamación. }\end{array}$ & $\begin{array}{c}\text { Análisis } \\
\text { de regresión } \\
\text { logística } \\
\text { (modelo } \\
\text { ANCOVA). }\end{array}$ & $\begin{array}{l}\text { La investigación } \\
\text { señala que del nivel } \\
\text { de información, de } \\
\text { experiencia y de la } \\
\text { importancia de la } \\
\text { situación, aumenta } \\
\text { la probabilidad de } \\
\text { una reclamación. }\end{array}$ \\
\hline $\begin{array}{c}\text { Stephens y } \\
\text { Gwinner (1998) }\end{array}$ & $\begin{array}{l}\text { Variable dependiente: } \\
\text { Acción de queja } \\
\text { y emociones. } \\
\text { Variables independientes: } \\
\text { Importancia de la situación } \\
\text { y probabilidad de éxito } \\
\text { de la reclamación. }\end{array}$ & $\begin{array}{c}\text { Análisis de } \\
\text { contenido } \\
\text { (muestreo de } \\
\text { conveniencia). }\end{array}$ & $\begin{array}{l}\text { La investigación muestra } \\
\text { que la probabilidad de } \\
\text { éxito de la reclamación } \\
\text { y la relevancia del } \\
\text { problema son } \\
\text { determinantes muy } \\
\text { relacionadas con la } \\
\text { acción de reclamación. }\end{array}$ \\
\hline $\begin{array}{c}\text { Maxham III y } \\
\text { Netemeyer } \\
\text { (2002) }\end{array}$ & $\begin{array}{l}\text { Variable dependiente: } \\
\text { Intención de recompra, } \\
\text { comentarios sobre } \\
\text { satisfacción. } \\
\text { Variables independientes: } \\
\text { Importancia del contexto, } \\
\text { probabilidad de éxito } \\
\text { de la reclamación } \\
\text { y sentimiento de culpa. }\end{array}$ & $\begin{array}{c}\text { Análisis } \\
\text { de regresión } \\
\text { ANCOVA } \\
\text { múltiple } \\
\text { (MANCOVA). }\end{array}$ & $\begin{array}{l}\text { La investigación } \\
\text { arroja que el contexto, } \\
\text { la probabilidad de éxito } \\
\text { de la reclamación y } \\
\text { el sentimiento de culpa } \\
\text { varían con respecto a la } \\
\text { cantidad de incidentes } \\
\text { de insatisfacción }\end{array}$ \\
\hline $\begin{array}{l}\text { Cruz y Galán } \\
\text { (2019) }\end{array}$ & $\begin{array}{l}\text { Variable dependiente: } \\
\text { Número de quejas } \\
\text { presentadas ante la } \\
\text { Procuraduría Federal } \\
\text { del Consumidor. } \\
\text { Variables independientes: } \\
\text { Nivel de ingreso, residencia } \\
\text { de los individuos (urbana o } \\
\text { rural), género y conciliación } \\
\text { entre individuo y empresa. }\end{array}$ & $\begin{array}{c}\text { Modelo } \\
\text { de datos de } \\
\text { panel estático. }\end{array}$ & $\begin{array}{l}\text { La investigación } \\
\text { encuentra que tanto el } \\
\text { producto interno bruto } \\
\text { por habitante, como la } \\
\text { probabilidad de éxito } \\
\text { de la queja y el } \\
\text { porcentaje de población } \\
\text { urbana tienen impacto } \\
\text { positivo sobre } \\
\text { el número de } \\
\text { reclamaciones. }\end{array}$ \\
\hline
\end{tabular}

Fuente: elaboración propia. 
Según Jin (2010), también existe la probabilidad de que el consumidor perciba que sus quejas son inútiles si siente que la compañía no valora sus inconformidades y, por lo tanto, no se quejará en el futuro. El autor también señala que puede esperarse que los consumidores no se quejen si creen que los costos de hacerlo serán más altos que los posibles beneficios. Por eso resulta muy importante resaltar la posible recompensa que puede obtener un quejoso como determinante de la decisión de inconformarse.

c) Ingreso de los consumidores

El ingreso es una variable que ha sido incluida para explicar el CQC. La literatura revisada (Barnhill, 1972; Stein, 1980; Kolodinsky, Murphy, Baehr y Lesser, 2005) reporta una relación positiva y significativa entre el nivel de ingreso de las personas que reclaman y el volumen de esta experiencia; es decir, se considera que los consumidores con menores ingresos son más vulnerables que los que tienen mayores ingresos. Esto obedece a que con un mayor nivel de ingreso, los consumidores poseen la capacidad de adquirir más satisfactores, por lo que sus episodios de compra aumentan, lo cual genera la posibilidad de incrementar las experiencias de insatisfacción y aumenta la propensión a inconformarse. Se afirma que las personas de altos ingresos son las que por lo común adquieren productos de alta calidad, mientras que las personas de bajos ingresos tendrán más dificultad para adquirirlos. De lo anterior se desprende que los consumidores de altos ingresos son más propensos a quejarse (Chinedu, Haron y Osman, 2017; Singh y Vigneswara, 2019). La mayoría de la literatura reporta una relación positiva entre el ingreso y el volumen de quejas presentado. La única excepción es un estudio de Hogarth, English y Sharma (2001), en el que se concluye que los consumidores de bajos ingresos tenían más probabilidades de utilizar agencias gubernamentales para ejercer sus reclamos.

d) Nivel educativo de los consumidores

De acuerdo con las investigaciones de Andreasen (1975), Smith y Cooper-Martin (1997) y Ringold (2005), los consumidores con menor educación formal parecen ser menos propensos a quejarse que los consumidores más educados. Esta relación puede deberse a varias razones: una es que las personas con mayor nivel de ingreso suelen tener niveles más altos de educación, por lo que tendrían un mayor conocimiento de los derechos de los consumidores; mientras que lo contrario sucedería con las personas de menores ingresos, que podrían tener menor conocimiento de estos derechos (Prasetyo, Hartoyo y Tinaprila, 2016). Los estudios de Andreasen (1975), Smith y Cooper-Martin (1997) y Ringold (2005) han señalado que los consumidores con menos educación tienen menos probabilidades de llevar a cabo acciones de reclamación ante agencias gubernamentales de protección a los derechos del consumidor. Sin embargo, dos estudios han concluido que los consumidores con menos educación son, en realidad, más propensos a ser denunciantes ante estas agencias del gobierno (Singh, 1989; Hogarth, English y Sharma, 2001). 
e) Género de los consumidores

El género es otra de las variables que ha presentado significancia estadística en el CQC (Hogarth, English y Sharma, 2001). Por ejemplo, para un estudio sobre la selección de servicios de hotelería en el Reino Unido, con un total de 104 encuestados, mayores de 55 años, y en el que las mujeres representaron $62.5 \%$ de la muestra, se encontró que ellas exhiben mayores expectativas al juzgar la calidad de los servicios (Callan y Bowman, 2000), lo que puede conducir a niveles más bajos de satisfacción en comparación con los hombres y, por ende, a incentivar un comportamiento de queja ante las agencias de protección a los derechos del consumidor. Podría ser que las mujeres tengan expectativas más altas en general (Hess, Ganesan y Klein, 2003), junto con puntajes de percepción más bajos en el momento de evaluar un producto o servicio (Kwok, Jusoh y Khalifah, 2016). El estudio de Laroche, Saad, Cleveland y Browne (2000) reveló que los hombres tienden a considerar menos información al evaluar las señales de servicio y tomar atajos en el procesamiento de la información, lo que puede resultar en una mayor satisfacción que las contrapartes femeninas, y de esta manera disminuir su comportamiento de queja.

Hess, Ganesan y Klein (2003) señalan que las mujeres tienen expectativas más altas que los hombres en sus episodios de consumo pero, al mismo tiempo, Kwok, Jusoh y Khalifah (2016) concluyen que, al respecto de los servicios, ellas asocian con mayor intensidad la satisfacción con la calidad. Aportes recientes a las diferencias de género en relación con el comportamiento de queja se pueden hallar en Wahyuni, Wardana, Yasa y Sukaatmadja (2020).

\section{Hipótesis y metodología econométrica}

Mediante la combinación de un corte transversal de los 32 estados que conforman la república mexicana durante un periodo de 11 años, se busca evaluar si el uso que hacen los consumidores desfavorecidos de los servicios de reclamación ante la PROFECO es una de las características dominantes propuestas en la literatura que se revisó. Se plantean las siguientes hipótesis:

H1: Puesto que los quejosos desarrollan mejores formas de quejarse debido a sus experiencias previas de queja exitosas, éstas tienen una asociación positiva con el CQC actual, pues incentiva su presentación.

H2: Haber obtenido algún tipo de reparación del daño, ya sea en forma de reembolso o de descuento, incentiva al quejoso a volver a presentar quejas. De la misma manera, los nuevos quejosos podrán sentirse más estimulados a presentar una reclamación si saben que sus quejas pueden tener éxito. Por esta razón se pretende probar que la percepción de éxito tiene una asociación positiva con el CQC.

$\mathrm{H3}$ : Cuando los consumidores tienen mayor ingreso, en general asociado con una mayor educación, tienden a conocer mejor sus derechos que los con- 
sumidores de bajos ingresos. Por eso se espera que el ingreso presente una relación positiva con el CQC.

H4: De acuerdo con las investigaciones de Callan y Bowman (2000) y Kolodinsky (1993), las mujeres muestran mayores expectativas en sus episodios de compra, en comparación con las expectativas de los hombres, por lo que podrían experimentar más insatisfacción. Por ello se pretende probar que las consumidoras con un mayor nivel educativo presentan una relación positiva con el CQC.

Para la comprobación estadística se desarrolla un modelo de datos de panel balanceado estático y después se incluye el efecto dinámico que impera en el comportamiento de queja de los consumidores mexicanos a través del método generalizado de momentos (MGM) que han desarrollado Arellano y Bond (1991).

El uso de datos de panel se ha incrementado, en general, en los estudios empíricos de problemas económicos y, en particular, en los estudios microeconómicos para la teoría del consumidor. De acuerdo con Hsiao (2003), se obtienen varios beneficios al utilizar la estructura de datos de panel. Entre los principales se encuentran los siguientes. 1) El control de la heterogeneidad: los datos de panel sugieren que los individuos, las empresas y los países son heterogéneos. 2) Los datos de panel proporcionan mayor cantidad de información, más variabilidad y menor colinealidad entre las variables y, por ende, más grados de libertad. 3) Los datos de panel pueden estudiar mejor la dinámica del ajuste. Deaton (1995) argumenta que, los datos de panel pueden relacionar las experiencias y el comportamiento del individuo en un momento determinado con otras experiencias y otro comportamiento en un momento diferente. 4) Los datos de panel pueden identificar y medir mejor los efectos que no se detectan en una sección transversal o en los datos de series temporales. 5) Los datos de micropanel reunidos acerca de los individuos, las empresas y los estados pueden medirse con mayor precisión que las variables similares medidas a nivel macro. El modelo básico para los datos de panel se encuentra determinado por la siguiente estructura:

$$
y_{i t}=\beta^{\prime} x_{i t}+\lambda_{i}+\gamma_{t}+v_{i t}, \quad(i=1, \ldots, N ; t=1, \ldots, T)
$$

Donde $B$ y $x_{i t}$ son vectores de dimensión $K$. El subíndice " $i$ " denota la sección cruzada de las unidades (entidades federativas) y el índice " $t$ " denota el periodo de tiempo. $\lambda_{i}$ y $\gamma_{t}$ son efectos individuales inobservables y efectos temporales, respectivamente. El término de error idiosincrático es $v_{i t}$. Si no se consideran los efectos individuales ni temporales, se parte del supuesto de que los coeficientes de intersección y de pendiente son los mismos para cada unidad que conforma el estudio. A este tipo de estructuras se las conoce como modelos agrupados. Si se considera que $\lambda_{i}$ es el parámetro a estimar, el modelo (1) se denomina modelo de efectos fijos, y el estimador de mínimos cuadrados ordinarios (MCO) de $\beta$ es conocido como el estimador intra-grupos (within o mínimos 
cuadrados con variable dicótoma). Por otro lado, si se considera que $\gamma_{t}$ es parte de la perturbación, el modelo (1) se conoce como modelo de efectos aleatorios y por lo general se emplea el estimador de mínimos cuadrados generalizados (MCG). A este grupo de modelos se les denomina datos de panel estáticos.

Aunque estos dos estimadores se usan mucho en estudios empíricos, el modelo (1) es restrictivo. Por ejemplo, si el efecto de tiempo denota un choque económico, entonces el modelo indica que tal impacto tiene efectos idénticos en todas las unidades de sección transversal, lo cual es poco probable que se mantenga en la práctica. Según Nerlove (2002), el comportamiento económico es dinámico en su naturaleza; por lo tanto, es importante tener en cuenta la dinámica en el modelo. Un enfoque común es usar la variable dependiente retrasada de $q_{i t}$ como variable explicativa:

$$
y_{i t}=\alpha y_{i, t-1}+\beta x_{i t}+\lambda_{i}+\gamma_{t}+v_{i t}, \quad(i=1, \ldots, N ; t=1, \ldots, T)
$$

Este tipo de modelo fue desarrollado por Balestra y Nerlove (1966) y se denomina modelo de datos de panel dinámico. Después de ese estudio, en la década de 1980, y como generalización del método de variables instrumentales, Arellano y Bond (1991) proponen el método generalizado de momentos (MGM), el cual consiste en calibrar el modelo combinando diversas variables instrumentales con respecto a un único vector numérico de coeficientes capaz de obtener correlaciones muestrales mínimas entre el término de error y cada uno de los instrumentos. El método puede ampliarse a la presencia de variables exógenas. De esta manera el MGM utiliza esas series en niveles y con retardos como instrumentos para la estimación de los parámetros del modelo.

De acuerdo con Sánchez y Viana (2017, p. 106), el criterio por el que se justifica elegir el método de estimación tiene que ver con la dificultad de tratar de forma adecuada el problema de la endogeneidad. ${ }^{1}$ Dosi (1988) señala que los datos de panel estáticos presentan una serie de carencias, entre las cuales destaca la incapacidad de tratar de manera apropiada el problema de la endogeneidad, lo que impide analizar la dependencia del pasado o del presente acumulativo. La mayoría de las veces el problema de endogeneidad es causado por la relación bidireccional entre el fenómeno que se quiere explicar y sus variables exógenas, y es el principal problema que se busca resolver con el MGM. Mediante la prueba de Sargan, se puede analizar la idoneidad de las variables instrumentales, por lo que es posible deducir si nos encontramos o no ante un modelo endógeno. Para capturar el impacto que puede tener la experiencia de quejas anteriores en las nuevas quejas, el modelo incluye como variable

De acuerdo con Rosales y Bonilla (2010), son varias las fuentes de endogeneidad, entre ellas: 1) Variable omitida. Este problema genera una dependencia condicional que conduce a estimadores sesgados e inconsistentes. 2) Simultaneidad. Se presenta cuando el planteamiento económico a describir contiene variables dependientes tratadas como independientes y relacionadas entre sí o que se determinan conjuntamente en un proceso. 3) Error de medición en las variables independientes. Surge por manipulación inadecuada de información o por una realización equivocada de encuestas. 4) Sesgo de selección. Aparece cuando los datos no son aleatorios para las variables independientes o explicativas, producto de errores en su recolección. 
independiente el rezago de la variable explicada; es decir, se busca utilizar una estructura dinámica autorregresiva que determine el impacto de la experiencia en el CQC con información anual para el caso mexicano.

\section{Análisis empírico}

Para comprender el CQC ante la agencia gubernamental de protección a los derechos del consumidor, se emplea un modelo de datos de panel para las 32 entidades federativas de México en el periodo de 2006 a 2017. La variable dependiente es el comportamiento de queja del consumidor $(q),{ }^{2}$ es decir, son las quejas totales presentadas a la Procuraduría Federal del Consumidor en cada entidad federativa del país. Las variables independientes, además del rezago de la variable endógena, son el producto interno bruto per cápita (y) como proxy del ingreso, el monto recuperado por queja como una variable proxy del éxito de queja $(m)$ y el nivel de educación de la población por sexo (neh, nem, nivel de educación para hombres y mujeres, respectivamente). ${ }^{3}$ Los datos se obtuvieron del Instituto Nacional de Transparencia y Acceso a la Información Pública y Protección de Datos Personales (INAI), del Instituto Nacional de Estadística y Geografía (INEGI) y del Consejo Nacional de Población (CONAPO).

Cabe mencionar que las quejas han sido agregadas en algunas entidades, pues hay estados que tienen varias delegaciones en diversas ciudades de su territorio, como el Estado de México, que tiene cinco delegaciones diferentes (Toluca, Naucalpan, Nezahualcóyotl, Tlalnepantla y Ecatepec). La PROFECO recibe y gestiona las quejas cuando el quejoso demuestra que el consumo por el que se está inconformando se llevó a cabo en el territorio de la entidad en la que presenta su queja. En este sentido, se considera conveniente subrayar que no todas las quejas que se presentan ante la procuraduría se convierten en un ingreso para los quejosos, sino que pasan por un proceso de varias etapas. Quedan fuera del análisis las quejas que los consumidores insatisfechos pudieron presentar en los respectivos departamentos de las empresas u otro tipo de quejas, como las que suelen hacerse en redes sociales u otros medios de comunicación. ${ }^{4}$

En la figura 1 se presenta la tasa media de crecimiento anual del total de las quejas en cada entidad federativa dividida en cuartiles. Las entidades que muestran las tasas más altas son Colima y Quintana Roo, con tasas de 6.8 y $9.5 \%$, respectivamente, mientras que las tasas más bajas, incluso negativas, se presentan en Baja California y Sinaloa con valores de -2.2 y $-2.1 \%$, respectivamente.

2 Los datos se adquirieron mediante una solicitud al INAI.

3 Los datos se obtuvieron de la Encuesta Nacional de Ocupación y Empleo (ENOE). La clasificación para el nivel de instrucción es: 1) primaria incompleta; 2) primaria completa; 3) secundaria completa y 4) educación media superior y superior. Todas las variables se clasificaron además por sexo: masculino y femenino.

4 No debe perderse de vista que un acto de consumo por el que un consumidor haya experimentado insatisfacción puede generar quejas en diversos medios y al mismo tiempo ser presentada en una procuraduría de defensa de derechos del consumidor por lo cual, para no tener una doble contabilización de las quejas se debe tener cuidado de diferenciarlas. 
Figura 1. Tasa media de crecimiento anual en el total de quejas de 2006 a 2017

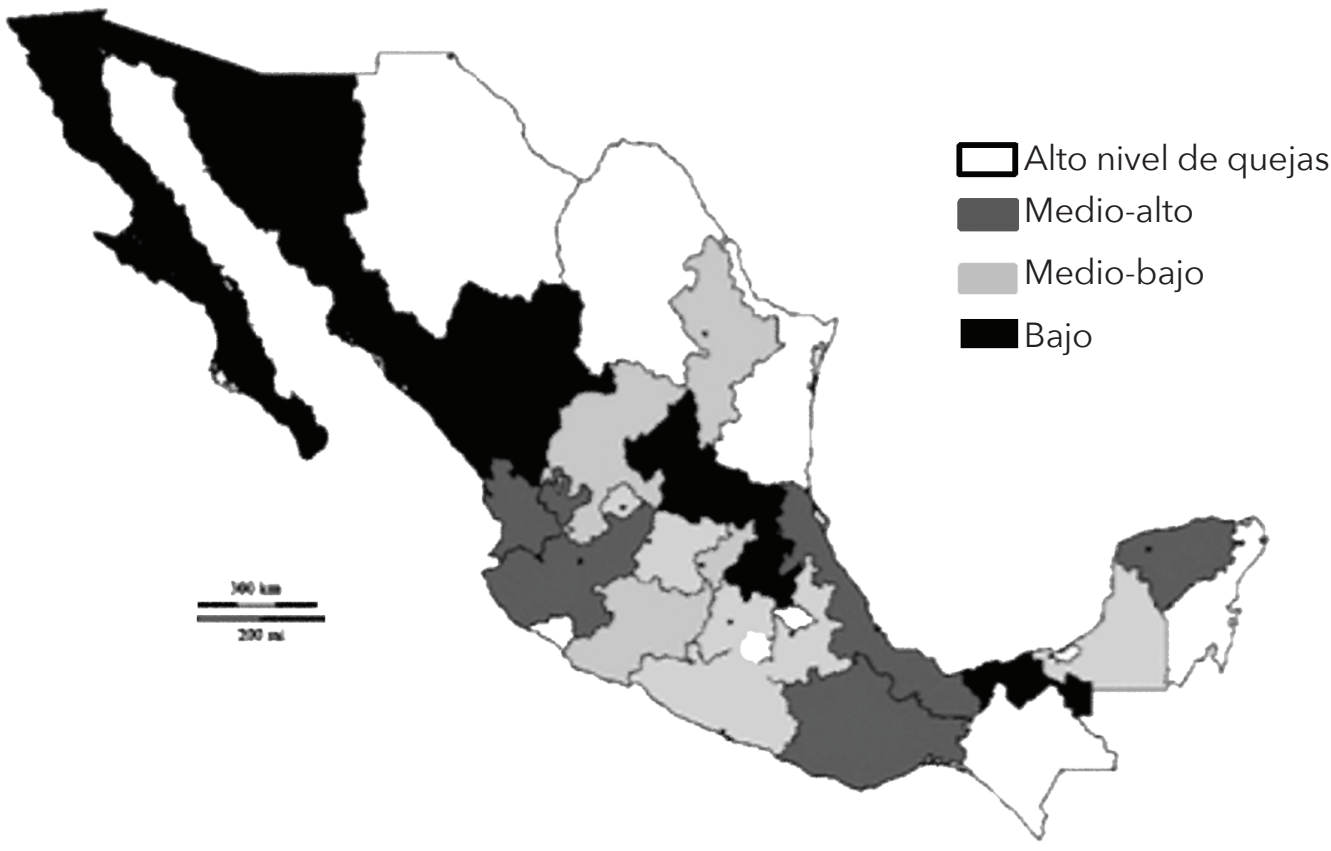

Fuente: elaboración propia con datos de la PROFECO (2018).

En lo que respecta a las variables independientes, en la figura 2 se presenta el producto interno bruto per cápita por entidad federativa (PIBPC) y el monto recuperado por queja (MRPQ). Las estadísticas muestran que los estados de la república con mayor ingreso por habitante son Ciudad de México, Nuevo León, Jalisco y el Estado de México, mientras que en promedio el monto que se recupera por cada inconformidad ante la PROFECO asciende a 7113 pesos. Se entiende esa cantidad como caso de éxito ante la presentación de una reclamación. Las entidades con mayores montos recuperados en promedio por queja son: Sinaloa con 15 278, Quintana Roo con 14 456, Nuevo León con 1349 y Campeche con 11908.

En la figura 3 se presentan los datos sobre el nivel educativo de los consumidores en las diferentes entidades federativas. Las variables corresponden a primaria incompleta (PINC), primaria completa (PCOM), secundaria completa (SCOM) y nivel medio superior y superior (MSYS). Los datos muestran que las entidades con mayor número de individuos con primaria inconclusa y con primaria completa - para el promedio de los años analizados en la muestra- son el Estado de México y Veracruz, mientras que el mayor número de individuos con secundaria concluida y con nivel medio superior y superior concluidos se concentra en el Estado de México y la Ciudad de México. 
Figura 2. Ingreso per cápita y monto recuperado por queja en las entidades federativas, 2006-2017

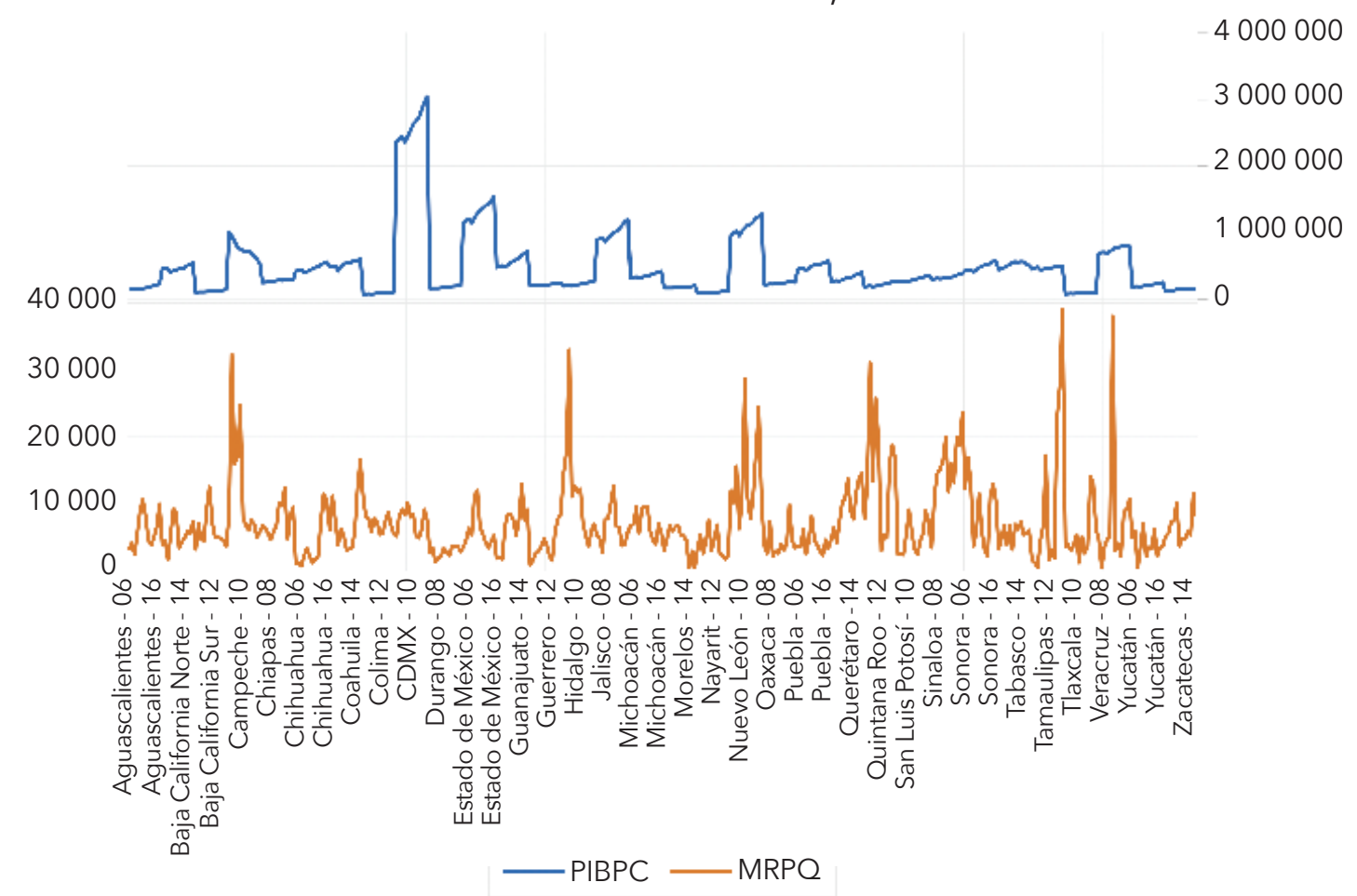

Fuente: elaboración propia con datos del INEGI (2019) y de la PROFECO (2018).

Figura 3. Nivel educativo de los consumidores por entidad federativa $5000000 \quad$ PINC

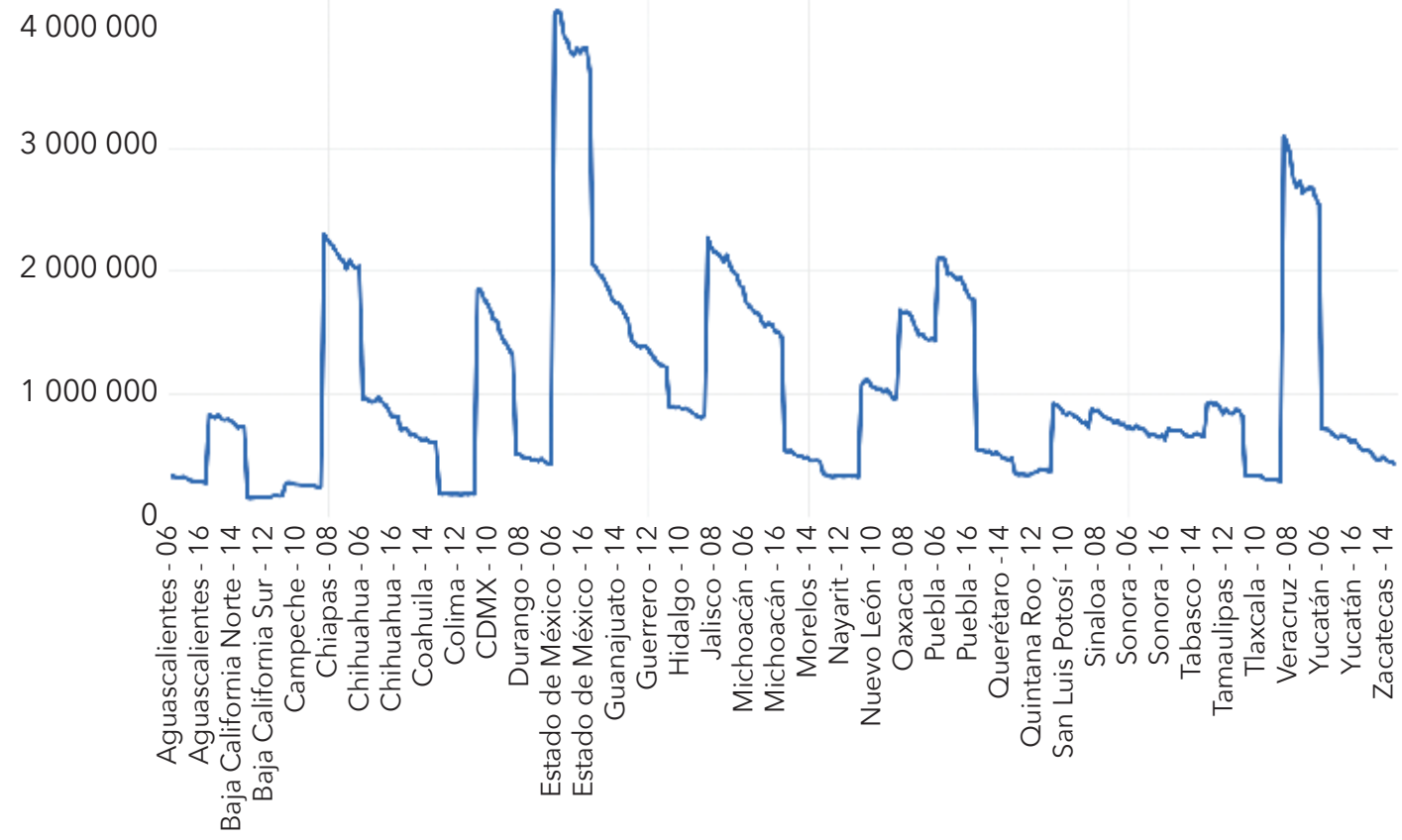




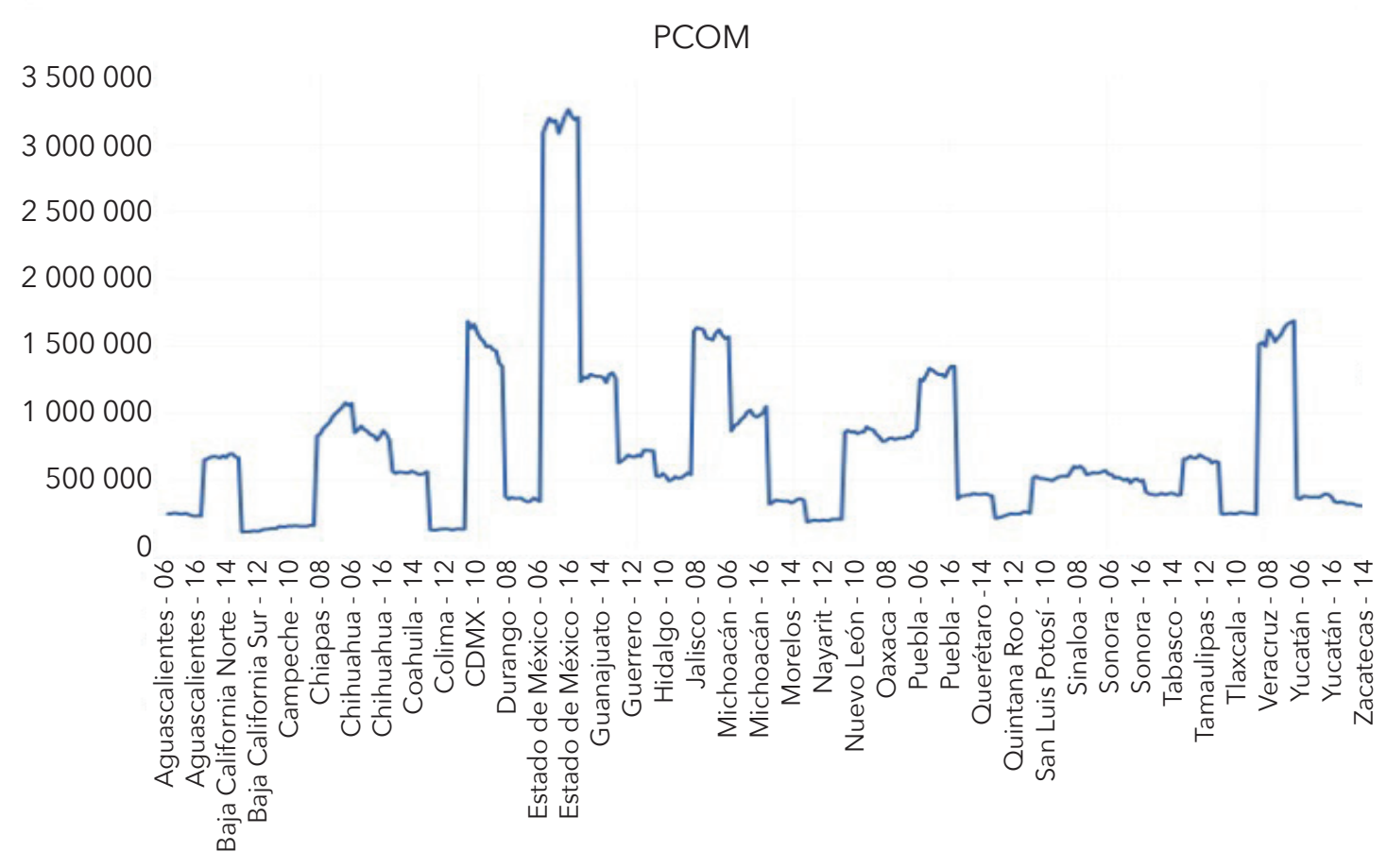

SCOM

6000000

5000000

4000000

3000000

2000000

1000000

0
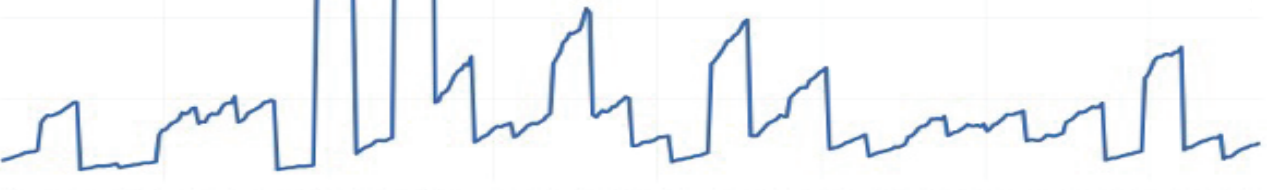

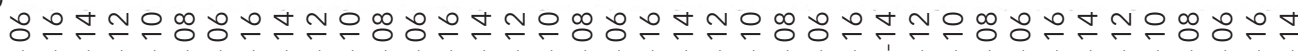

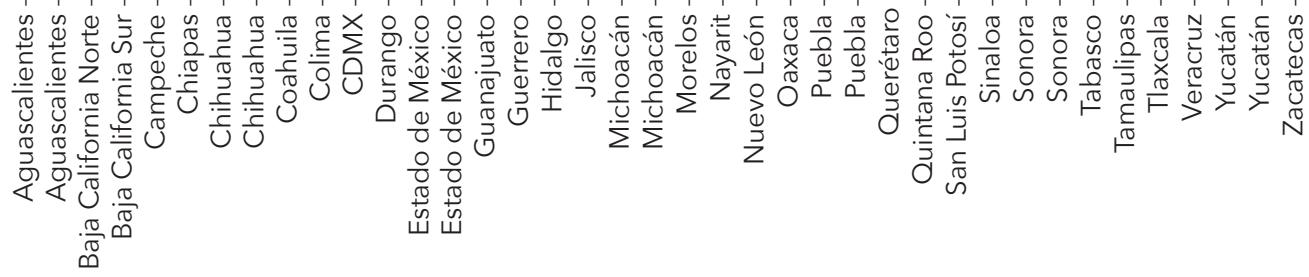


MSYS

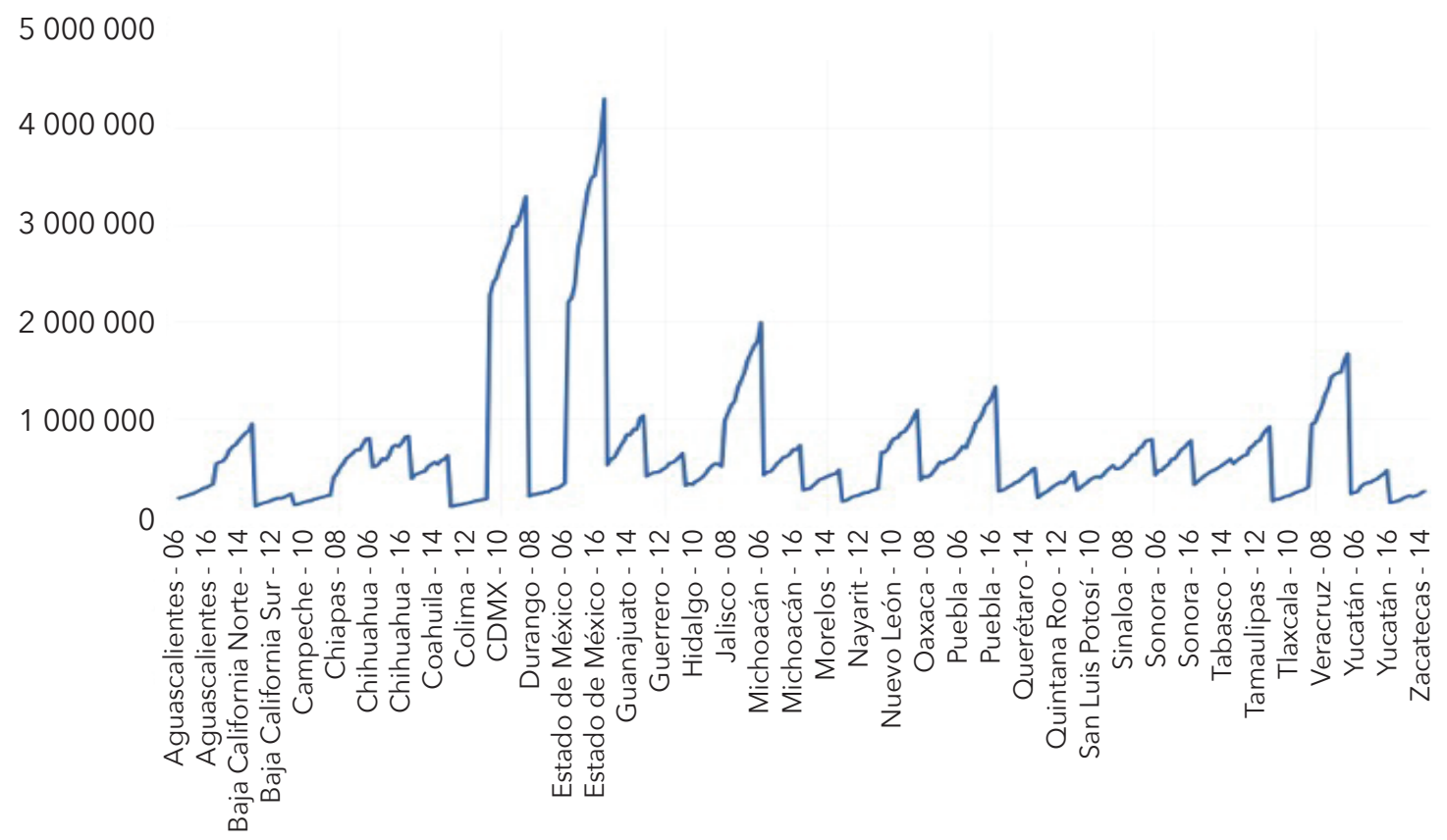

Fuente: elaboración propia con datos del INEGI (2019).

Hay que mencionar que el género de los consumidores puede representar una característica diferencial en el comportamiento de queja, como demuestra Kolodinsky (1993): empleando un modelo de ecuaciones simultáneas para explicar el comportamiento de queja de los consumidores estadounidenses de servicios médicos, encuentra evidencia de que la variable género (mujer) presenta una fuerte significancia estadística.

\section{Resultados}

Una vez identificadas las variables que determinan el comportamiento de queja de los consumidores, se busca estimar un modelo de datos de panel. En la primera etapa se desarrolla una estructura estática que capture el comportamiento que exhiben los habitantes de las diferentes entidades federativas. El modelo propuesto se representa de la siguiente manera:

$$
q_{i t}=\alpha+\beta X_{i t}+v_{i t}, \quad(i=1, \ldots, N ; t=1, \ldots, T)
$$

La expresión “i” denota las entidades federativas y " $t$ " el periodo 2006 a 2017; $q_{i t}$ representa el CQC, definido por las quejas totales presentadas a la 
PROFECO, mientras que $\alpha$ es un escalar, $\beta$ es una matriz de $K x 1$, y $X_{i t}$ representa las variables exógenas, donde se encuentra el ingreso $(y)$ representado por el PIBPC; el éxito de la queja es $(m)$ capturado por el monto recuperado por queja y el nivel educativo de la población por sexo (neh y neh): nivel de educación para hombres y mujeres, respectivamente. También se utiliza un componente de error unidireccional para las perturbaciones $V_{i t}=\lambda_{i}+U_{i t}$, donde $\lambda_{i}$ representa el efecto específico individual inobservable y $U_{i t}$ denota las perturbaciones restantes. Después se desarrollaron pruebas de raíz unitaria con datos de panel para identificar el grado de integración de las variables y evitar así el problema de la regresión espuria. Todas las variables presentan estacionariedad en las vares transformadas como tasas de crecimiento (véase tabla 2).

Tabla 2. Pruebas de estacionariedad (raíz unitaria)

\begin{tabular}{|c|c|c|c|c|c|c|c|}
\hline Variables & Modelo & LLC stat & Prob & ADF stat & Prob & PP stat & Prob \\
\hline$D L q_{i t}$ & $\begin{array}{c}C \\
C_{y} T^{2}\end{array}$ & $\begin{array}{l}-6.56 \\
-6.25\end{array}$ & $\begin{array}{l}0.000 \\
0.000\end{array}$ & $\begin{array}{r}138.51 \\
94.55 \\
\end{array}$ & $\begin{array}{l}0.000 \\
0.007\end{array}$ & $\begin{array}{l}463.86 \\
323.82 \\
\end{array}$ & $\begin{array}{l}0.000 \\
0.000\end{array}$ \\
\hline$D L y_{i t}$ & $\begin{array}{c}C \\
\text { Cy }\end{array}$ & $\begin{array}{r}-5.895 \\
-12.421 \\
\end{array}$ & $\begin{array}{l}0.000 \\
0.000\end{array}$ & $\begin{array}{r}98.42 \\
118.41 \\
\end{array}$ & $\begin{array}{l}0.004 \\
0.000 \\
\end{array}$ & $\begin{array}{l}212.08 \\
282.10 \\
\end{array}$ & $\begin{array}{l}0.000 \\
0.000\end{array}$ \\
\hline$D L m_{i t}$ & $\begin{array}{c}C \\
\text { Cy }\end{array}$ & $\begin{array}{l}-11.29 \\
-12.65 \\
\end{array}$ & $\begin{array}{l}0.000 \\
0.000 \\
\end{array}$ & $\begin{array}{l}169.72 \\
123.72 \\
\end{array}$ & $\begin{array}{l}0.004 \\
0.000 \\
\end{array}$ & $\begin{array}{l}381.71 \\
301.42 \\
\end{array}$ & $\begin{array}{l}0.000 \\
0.000\end{array}$ \\
\hline DLHPINC $_{i t}$ & $\begin{array}{c}C \\
\text { Cy }\end{array}$ & $\begin{array}{l}-14.71 \\
-14.41 \\
\end{array}$ & $\begin{array}{l}0.000 \\
0.000 \\
\end{array}$ & $\begin{array}{l}200.35 \\
138.78 \\
\end{array}$ & $\begin{array}{l}0.000 \\
0.002 \\
\end{array}$ & $\begin{array}{l}377.27 \\
306.39 \\
\end{array}$ & $\begin{array}{l}0.000 \\
0.000\end{array}$ \\
\hline DLHPCOM $_{i}$ & $\begin{array}{c}C \\
\text { CyT }\end{array}$ & $\begin{array}{l}-9.38 \\
-8.95 \\
\end{array}$ & $\begin{array}{l}0.000 \\
0.000 \\
\end{array}$ & $\begin{array}{l}166.93 \\
112.42 \\
\end{array}$ & $\begin{array}{l}0.000 \\
0.002 \\
\end{array}$ & $\begin{array}{l}320.22 \\
294.41 \\
\end{array}$ & $\begin{array}{l}0.000 \\
0.000\end{array}$ \\
\hline DLHSCOM $_{i t}$ & $\begin{array}{c}C \\
\text { Cy }\end{array}$ & $\begin{array}{l}-11.35 \\
-16.11 \\
\end{array}$ & $\begin{array}{l}0.000 \\
0.000 \\
\end{array}$ & $\begin{array}{l}144.81 \\
148.28 \\
\end{array}$ & $\begin{array}{l}0.000 \\
0.000 \\
\end{array}$ & $\begin{array}{l}223.23 \\
271.24 \\
\end{array}$ & $\begin{array}{l}0.000 \\
0.000\end{array}$ \\
\hline DLHMSYS $_{i t}$ & $\begin{array}{c}C \\
C y T\end{array}$ & $\begin{array}{l}-7.31 \\
-6.61\end{array}$ & $\begin{array}{l}0.000 \\
0.000 \\
\end{array}$ & $\begin{array}{r}136.45 \\
93.94 \\
\end{array}$ & $\begin{array}{l}0.000 \\
0.000 \\
\end{array}$ & $\begin{array}{l}309.57 \\
272.62\end{array}$ & $\begin{array}{l}0.000 \\
0.000\end{array}$ \\
\hline DLMPINC $_{i t}$ & $\begin{array}{c}C \\
\text { CyT }\end{array}$ & $\begin{array}{l}-6.83 \\
-4.59 \\
\end{array}$ & $\begin{array}{l}0.000 \\
0.000 \\
\end{array}$ & $\begin{array}{r}139.41 \\
86.08 \\
\end{array}$ & $\begin{array}{l}0.000 \\
0.034 \\
\end{array}$ & $\begin{array}{l}260.83 \\
207.93 \\
\end{array}$ & $\begin{array}{l}0.000 \\
0.000\end{array}$ \\
\hline$D_{M L M P C O}$ & $\begin{array}{c}C \\
C y T\end{array}$ & $\begin{array}{r}-8.81 \\
-11.11 \\
\end{array}$ & $\begin{array}{l}0.000 \\
0.000 \\
\end{array}$ & $\begin{array}{l}149.77 \\
108.04 \\
\end{array}$ & $\begin{array}{l}0.000 \\
0.000 \\
\end{array}$ & $\begin{array}{l}306.35 \\
267.78 \\
\end{array}$ & $\begin{array}{l}0.000 \\
0.000\end{array}$ \\
\hline DLMSCOM $_{i}$ & $\begin{array}{c}C \\
\text { Cy }{ }^{\top}\end{array}$ & $\begin{array}{l}-9.31 \\
-9.34 \\
\end{array}$ & $\begin{array}{l}0.000 \\
0.000 \\
\end{array}$ & $\begin{array}{l}160.59 \\
124.85 \\
\end{array}$ & $\begin{array}{l}0.000 \\
0.000 \\
\end{array}$ & $\begin{array}{l}318.31 \\
303.11 \\
\end{array}$ & $\begin{array}{l}0.000 \\
0.000\end{array}$ \\
\hline DLMMSYS $_{i t}$ & $\begin{array}{c}C \\
\text { CyT }^{\top}\end{array}$ & $\begin{array}{l}-5.67 \\
-5.24\end{array}$ & $\begin{array}{l}0.000 \\
0.000\end{array}$ & $\begin{array}{r}127.55 \\
97.37 \\
\end{array}$ & $\begin{array}{l}0.000 \\
0.004\end{array}$ & $\begin{array}{l}302.71 \\
280.77\end{array}$ & $\begin{array}{l}0.000 \\
0.000\end{array}$ \\
\hline
\end{tabular}

Nota:

$\mathrm{DL}=$ representa la diferencia logarítmica aplicada a todas las variables.

LLC = Levin, Lin y Chu, la hipótesis nula: raíz unitaria (asume procesos de raíz unitaria comunes).

ADF = Dickey-Fuller aumentada, la hipótesis nula: raíz unitaria.

$\mathrm{PP}=$ Phillips Perron, la hipótesis nula: raíz unitaria (asume procesos de raíz unitaria individuales).

$\mathrm{C}=$ constante.

C y $T=$ constante y tendencia.

Fuente: elaboración propia. 
Los resultados de la tabla 3 presentan los coeficientes de los tres modelos estimados. El primer modelo corresponde a una estructura de datos agrupados. El segundo modelo de efectos fijos (within) mide la variación en el tiempo para cada entidad federativa en específico, mediante la inclusión de una variable dicótoma para cada estado de la república mexicana y para cada año. La tercera estructura corresponde a un modelo de efectos aleatorios que, a diferencia del modelo previo, evita la pérdida de grados de libertad, pues el término $\lambda_{i}$ de la ecuación (1) es aleatorio. Las primeras dos estructuras se estiman mediante MCO y la tercera, mediante mínimos cuadrados generalizados estimados (MCGE).

Tabla 3. Modelo de datos de panel estático para el COC para las entidades federativas

\begin{tabular}{|c|c|c|c|c|}
\hline & $D L q_{i t}$ & $D L q_{i t}$ & $D L q_{i t}$ & $D L q_{i t}$ \\
\hline $\begin{array}{l}\text { Variables } \\
\text { explicativas }\end{array}$ & $\begin{array}{l}\text { Modelo [1] } \\
\text { agrupado }\end{array}$ & $\begin{array}{l}\text { Modelo [2] E. } \\
\text { fijos (within) }\end{array}$ & $\begin{array}{c}\text { Modelo [3] E. } \\
\text { aleatorios }\end{array}$ & $\begin{array}{l}\text { Modelo [4] E. } \\
\text { fijos (vcovHC) }\end{array}$ \\
\hline$C$ & $\begin{array}{c}-0.119 \\
{[-3.162]^{*+*}}\end{array}$ & $\begin{array}{c}-0.173 \\
{[-2.94]^{+*+}}\end{array}$ & $\begin{array}{c}-0.121 \\
{[-2.876]^{*+*}}\end{array}$ & $\begin{array}{l}-0.173 \\
{[0.50]}\end{array}$ \\
\hline$D L y_{i t}$ & $\begin{array}{c}0.347 \\
{[0.926]}\end{array}$ & $\begin{array}{c}0.015 \\
{[0.023]}\end{array}$ & $\begin{array}{c}0.348 \\
{[0.804]}\end{array}$ & $\begin{array}{c}0.015 \\
{[0.031]}\end{array}$ \\
\hline$D L m_{i t}$ & $\begin{array}{c}-0.148 \\
{[-7.357]^{+* * *}}\end{array}$ & $\begin{array}{c}-0.155 \\
{[-7.572]^{+* * *}}\end{array}$ & $\begin{array}{c}-0.148 \\
{[-7.413]^{+*+*}}\end{array}$ & $\begin{array}{c}-0.155 \\
{[-3.90]^{+* *}}\end{array}$ \\
\hline$D^{D L H P I N C} C_{i t}$ & $\begin{array}{c}1.058 \\
{[1.903]^{*}} \\
\end{array}$ & $\begin{array}{c}1.615 \\
{[2.307]^{* *}} \\
\end{array}$ & $\begin{array}{c}1.058 \\
{[1.829]^{*}} \\
\end{array}$ & $\begin{array}{c}1.615 \\
{[2.14]^{* *}}\end{array}$ \\
\hline$D_{L H P C O M_{i t}}$ & $\begin{array}{c}0.677 \\
{[1.421]}\end{array}$ & $\begin{array}{c}1.168 \\
{[1.979]^{*}}\end{array}$ & $\begin{array}{l}0.677 \\
{[1.36]}\end{array}$ & $\begin{array}{c}1.168 \\
{[1.923]^{*}}\end{array}$ \\
\hline$D^{D L H S C O M}{ }_{i t}$ & $\begin{array}{c}0.638 \\
{[1.392]}\end{array}$ & $\begin{array}{c}1.177 \\
{[1.971]^{*}}\end{array}$ & $\begin{array}{c}0.638 \\
{[1.311]}\end{array}$ & $\begin{array}{c}1.177 \\
{[2.413]^{* *}}\end{array}$ \\
\hline$D_{L H M S Y S}{ }_{i t}$ & $\begin{array}{c}0.136 \\
{[0.313]}\end{array}$ & $\begin{array}{c}0.541 \\
{[1.044]}\end{array}$ & $\begin{array}{c}0.136 \\
{[0.297]}\end{array}$ & $\begin{array}{c}0.541 \\
{[1.044]}\end{array}$ \\
\hline$D L M P I N C_{i t}$ & $\begin{array}{l}-0.0104 \\
{[-0.018]}\end{array}$ & $\begin{array}{c}0.312 \\
{[0.417]}\end{array}$ & $\begin{array}{c}-0.011 \\
{[-0.017]}\end{array}$ & $\begin{array}{c}0.312 \\
{[0.417]}\end{array}$ \\
\hline DLMPCOM & $\begin{array}{c}0.012 \\
{[0.024]}\end{array}$ & $\begin{array}{c}0.373 \\
{[0.575]}\end{array}$ & $\begin{array}{c}0.012 \\
{[0.023]}\end{array}$ & $\begin{array}{c}0.373 \\
{[0.575]}\end{array}$ \\
\hline$D^{D L M S C O M}{ }_{i t}$ & $\begin{array}{c}1.256 \\
{[2.195]^{+*}}\end{array}$ & $\begin{array}{c}1.792 \\
{[2.544]^{*+*}}\end{array}$ & $\begin{array}{c}1.256 \\
{[2.122]^{+*}}\end{array}$ & $\begin{array}{c}1.792 \\
{[2.07]^{* *}}\end{array}$ \\
\hline DLMMSYS ${ }_{i t}$ & $\begin{array}{c}0.901 \\
{[2.012]^{*}}\end{array}$ & $\begin{array}{c}1.173 \\
{[2.177]^{* *}}\end{array}$ & $\begin{array}{c}0.901 \\
{[1.945]^{*}}\end{array}$ & $\begin{array}{c}1.173 \\
{[1.89]^{*}}\end{array}$ \\
\hline $\begin{array}{c}R^{2} \\
F-\text { stat } \\
D . W .\end{array}$ & \begin{tabular}{|c}
0.178 \\
$7.42[0.000]$ \\
2.31
\end{tabular} & $\begin{array}{c}0.251 \\
2.13[0.002] \\
2.38\end{array}$ & $\begin{array}{c}0.178 \\
7.42[0.000] \\
2.31\end{array}$ & $\begin{array}{c}0.251 \\
2.13[0.002] \\
2.38\end{array}$ \\
\hline
\end{tabular}

Nota:

$\mathrm{DL}=$ representa la diferencia logarítmica aplicada a todas las variables.

vcovHC = matriz de varianzas covarianzas consistente con heterocedasticidad

Los resultados entre corchetes representan los valores estadísticos de los estimadores.

${ }^{* \star *}=$ significancia al nivel 0.01

${ }^{\star *}=$ significancia al nivel 0.05

* = significancia al nivel 0.10

Fuente: elaboración propia. 
Para determinar el modelo que capture las características esenciales del comportamiento de queja del consumidor mexicano, se recurrió a la prueba F-restrictiva, que permite discernir entre un modelo agrupado y otro de efectos fijos. El resultado de la prueba muestra un valor estadístico de 1.978, con un

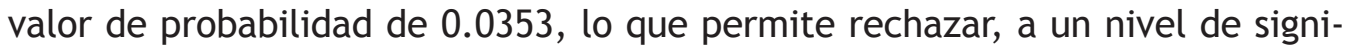
ficancia de $5 \%$, la hipótesis nula de que todos los coeficientes de las variables dicótomas fijas no tienen influencia. Esto demuestra que el modelo de efectos fijos (within) exhibe un mejor ajuste para explicar el fenómeno analizado, ya que considera el efecto que genera cada uno de los once años analizados en la muestra. Luego se desarrolló una prueba de multiplicadores lagrangianos de Breusch-Pagan para efectos aleatorios, con el fin de determinar la idoneidad entre un modelo agrupado y otro de efectos aleatorios. El estadístico $X^{2}$ muestra un valor de 16.05 , con un valor de probabilidad de 0.001 , lo que permite rechazar la hipótesis nula de no existencia de efectos de panel aleatorios; en consecuencia, se prefiere un modelo de efectos aleatorios sobre el modelo de efectos agrupados.

Una vez que el modelo de efectos fijos y el modelo de efectos aleatorios demostraron ser superiores al modelo de efectos agrupados, se realizó una prueba de Hausman para decidir entre el modelo de efectos fijos y el modelo de efectos aleatorios. El estadístico $X^{2}$ es de 37.2 con un valor de probabilidad de 0.0007 , lo cual permite rechazar la hipótesis nula de que los errores no están correlacionados con los regresores. En consecuencia, existe evidencia de la superioridad del modelo de efectos fijos (within). De acuerdo con Baltagi (2005), una vez identificada la estructura óptima, se debe comprobar la posible existencia de correlación serial. Se desarrolló la prueba Breusch-Godfrey/Wooldridge para la correlación serial en modelos de panel y el estadístico; $X^{2}$ indicó un valor de 137 , con una probabilidad de 0.000 , lo que señala la correlación serial en los términos idiosincráticos. Además, se estimó una prueba de heterocedasticidad de Breusch-Pagan con un valor de 338 y un valor de significancia de 0.000 , por lo cual también se identifica que el modelo exhibe problemas de varianzas desiguales.

Por último, ante la presencia de autocorrelación serial y de heterocedasticidad, se utilizó una matriz de varianzas covarianzas robustas ${ }^{5}$ para considerar las problemáticas descritas. Utilizando la especificación de Arellano (1987), se castigaron los errores estándar y se obtuvieron los siguientes resultados. La ecuación 4 no muestra significancia estadística para el ingreso. Sin embargo, esto puede deberse a un efecto rezago, es decir que el ingreso anterior sea quien impacte en el CQC, por lo cual es necesario establecer un modelo de datos de panel dinámico para corroborar si éste es el caso. Con respecto al efecto causado por el éxito de la queja, se observa una relación inversa; es decir, a medida que el monto de recuperación por la queja es menor, el consumidor se verá incentivado a realizar reclamaciones, mientras que a mayor

5 De acuerdo con Zeileis (2004), la matriz de varianzas covarianzas consistente con heterocedasticidad contiene tres especificaciones:

i) "white1": para heterocedasticidad general, pero sin correlación serial (efectos aleatorios).

ii) "white2": es "white1" restringido a una variación común dentro de los grupos (efectos aleatorios).

iii) "arellano": tanto heterocedasticidad como correlación serial (efectos fijos). 
monto reclamado, se presenta una disminución en las inconformidades. Eso puede atribuirse a que el consumidor mexicano experimenta una renuencia a quejarse por altos montos al no ver prosperar la queja, tal como lo establece Jin (2010). En relación con la variable que representa un mayor nivel educativo, los resultados muestran que los hombres con primaria incompleta y completa, al igual que con secundaria completa, son los que más se quejan. Los resultados coinciden con lo que señalan Singh (1989), Hogarth, English y Sharma (2001) y Laroche, Saad, Cleveland y Browne (2000). Por otro lado, los coeficientes que capturan el nivel educativo de las mujeres revelan que a mayor preparación existe un incremento en la propensión a inconformarse, lo que es consistente con lo que demuestran Callan y Bowman (2000), Hess, Klein y Ganesan (2003), Kwok, Jusoh y Khalifah (2016).

A continuación, como una segunda etapa, se presenta la especificación de un modelo de datos de panel dinámico, mediante el MGM, que permita tratar de forma adecuada el problema de la endogeneidad y que capture el impacto de las experiencias de quejas previas en las inconformidades actuales. Se considera la siguiente estructura:

$$
q_{i t}=\alpha q_{i(t-1)}+\beta X_{i t}+\lambda_{i}+\gamma_{t}+v_{i t}
$$

Donde $q_{i t}$ es el logaritmo de las quejas en cada una de las entidades federativas al final de cada año de 2006 a 2017. El vector $X_{i t}$ contiene las variables de ingreso $(y)$, el éxito de queja $(m)$ y el nivel de educación para hombres y mujeres (neh y nem). La especificación también contiene un efecto $\gamma_{t}$, que es común a todas las entidades federativas, y un efecto específico para cada entidad, que es permanente pero inobservable. También contiene un término de error $v_{i t}$.

En la tabla 4 se reportan las estimaciones del MGM. Se comenzó con una estructura de hasta dos rezagos por cada variable exógena. Sin embargo, el segundo rezago de ambas variables resultó no significativo. La primera y la segunda columna muestran la especificación dinámica más general para el mecanismo de un paso y de dos pasos, respectivamente. ${ }^{6}$ El primero de ellos utiliza sólo la matriz de pesos homocedástica para la estimación, mientras que la segunda emplea para la estimación la matriz de pesos heterocedástica. También se debe subrayar que ni los estadísticos de robustez $m_{2}$ (prueba de correlación serial de Arellano-Bond, 1991) ni la prueba de dos etapas de Sargan son rechazados, lo cual quiere decir que todas las variables instrumentales incluidas son válidas, pues los errores no están correlacionados de manera serial.

Los cuatro modelos con mecanismo de dos pasos muestran que la experiencia previa de inconformidad ante la agencia gubernamental afecta la actitud y el comportamiento del consumidor mexicano con respecto al número de quejas actuales. Esto demuestra que el consumidor desarrolla una propensión hacia la queja como resultado de sus experiencias previas, que pueden estar asociadas con el conocimiento de nuevos métodos o vías para externar su insatisfacción.

6 De acuerdo con Arellano y Bond (1991), los errores asintóticos estándar asociados a las estimaciones de dos etapas son, por lo general, alrededor de $30 \%$ más bajos que aquellos asociados con las estimaciones de un solo paso. 


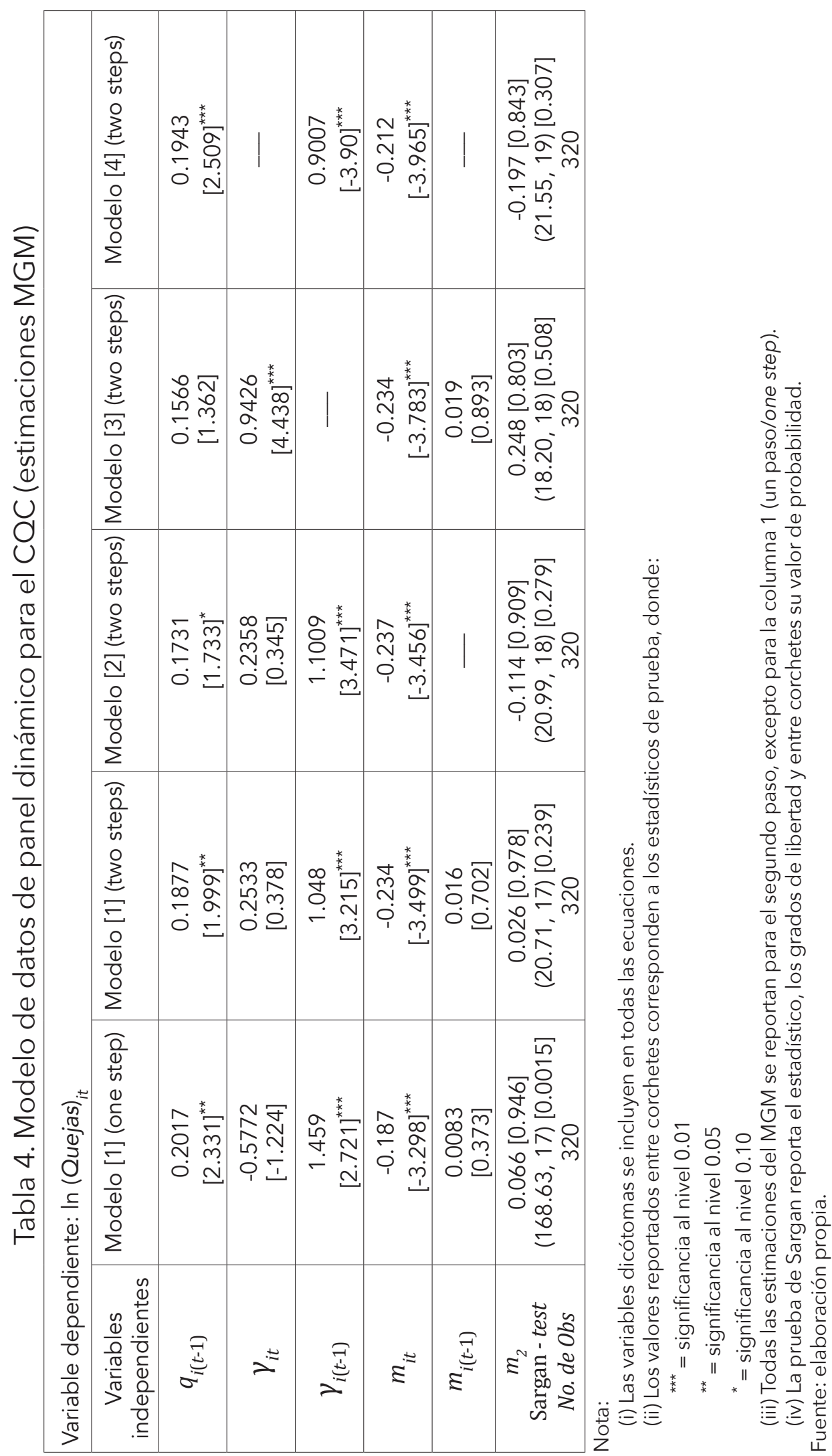


Estos resultados coinciden con los que encontraron Ursic (1985), Martin (1991), Singh y Wilkes (1996) y Kim, Kim, Im y Shin (2003).

A diferencia del modelo de datos de panel estático, en el que no se pudo observar ningún efecto del ingreso en el número de quejas, en los modelos dinámicos se puede identificar que existe una relación positiva y significativa entre el nivel de ingreso de las personas con un periodo de rezago y el volumen de las quejas. Esto muestra que existe un comportamiento dinámico en el patrón de quejas de los consumidores mexicanos. Y que el aumento en el ingreso produce un incremento de episodios de compra que, a su vez, estimulan la posibilidad de producir más experiencias de insatisfacción y presentación de quejas. Esta evidencia concuerda con los resultados de Volkov, Harker y Harker (2005), Garrett y Toumanoff (2010), Chinedu, Haron y Osman (2017) y Singh y Vigneswara (2019).

Sobre la probabilidad de éxito, los resultados coinciden con el modelo estático en cuanto al signo y a la significancia estadística, aunque el coeficiente es un poco mayor. Como ya se mencionó, la relación muestra que a medida que el monto de recuperación derivado de la queja es mayor, se desincentiva el comportamiento de queja. Este resultado obedecería a que el procedimiento de la queja puede llegar a demorar demasiado y por ende desmotivar al quejoso para llegar al final del procedimiento. En cambio, la compensación de los montos bajos puede resultar más rápida y menos compleja. Cabe mencionar que en el modelo dinámico también se incluyeron las variables correspondientes al nivel de educación de los consumidores, clasificados por hombre y mujer (neh y nem). Sin embargo, los coeficientes no presentaron significancia estadística.

\section{Conclusiones}

Esta investigación identifica los determinantes que inciden en el comportamiento de queja de los consumidores mexicanos en el periodo 2006-2017. El análisis de los datos recolectados y la estimación de modelos robustos aportan información relevante sobre una variable poco estudiada en México. Además, se desarrolla una metodología para determinar el comportamiento dinámico del fenómeno, lo que constituye su principal contribución.

Mediante la estimación de modelos de paneles estáticos y dinámicos, se comprobaron las hipótesis planteadas sobre el comportamiento de quejas presentadas ante la PROFECO. Éste fue cuantificado como el total de las quejas anuales presentadas en las entidades federativas del país. Los principales hallazgos pueden resumirse de la siguiente manera:

1) La experiencia previa de la queja tiene correlación positiva con el CQC debido a que los consumidores aprenden y mejoran sus métodos de queja por medio de la práctica. 2) En la medida en que el monto de recuperación por queja presentada aumenta, el consumidor se verá desincentivado a realizar reclamaciones. 3) A mayor ingreso per cápita de los consumidores, mayor es el estímulo para presentar quejas ante la PROFECO, pues este segmento de consumidores exhibe mayor conocimiento y tiene más conciencia sobre los derechos 
del consumidor. Lo anterior se deriva de que las personas con mayor nivel de ingreso suelen tener niveles educativos más altos; además de que, por lo común, adquieren productos de alta calidad. 4) Las mujeres consumidoras con mayores niveles educativos exhiben una relación positiva sobre las quejas presentadas ante la PROFECO, pues por lo general muestran mayores expectativas al juzgar la calidad de los servicios, es decir, asocian la satisfacción con la calidad con mayor intensidad que los hombres. En el caso de los hombres, la evidencia indica que la población con menor nivel educativo es la que exhibe una mayor propensión a presentar reclamaciones.

\section{Referencias}

Abney, A., Pelletier, M., Ford, T., y Horky, A. (2017). I hate your brand: adaptive service recovery strategies on Twitter. Journal of Services Marketing, 31(3), 281-294.

Andreasen, A. (1975). The Disadvantaged Consumer. Nueva York: The Free Press.

Arellano, M. (1987). Computing robust standard errors for within-groups estimators. Oxford Bulletin of Economics and Statistics, 49(4), 431-434.

Arellano, M., y Bond, S. (1991). Some tests of specification for panel data: Monte Carlo evidence and an application to employment equations. Review of Economic Studies (58), 277-297. doi: https: / / doi.org/10.2307/2297968

Ashraf, T., Waseem, S., Rizwan, M., Ahmed, D., y Nazeer, H. (2013). Determinants of consumer complaining behavior: a study of Pakistan. International Journal of Learning and Development, 3(6), 121-139. doi: https://doi. org/10.5296/ijld.v3i6.6209

Balestra, P., y Nerlove, M. (1966). Pooling cross-section and time-series data in the estimation of a dynamic model: the demand for natural gas. Econometrica (34), 585-612. doi: https: / / doi.org/10.2307/1909771

Baltagi, B. (2005) Econometric Analysis of Panel Data. Nueva York: John Wiley \& Sons Inc.

Barnhill, J. (1972). Market injustice: the case of the disadvantaged consumer. Journal of Consumer Affairs, 6(1), 78-83.

Berry, L., Seiders, K., y Grewal, D. (2002). Understanding service convenience. Journal of Marketing (66), 1-17. doi: https://doi.org/10.1509/ jmkg.66.3.1.18505

Callan, R., y Bowman, L. (2000). Selecting a hotel and determining salient quality attributes: a preliminary study of mature British travelers. International Journal of Tourism Research, 2(2), 97-118. doi: https://doi.org/10.1002/ (SICI)1522-1970(200003/04)2:2<97::AID-JTR190>3.0.CO;2-1

Chinedu, A., Haron, S., y Osman, S. (2017). Predictors of complaint. Behavior among Mobile Telecommunications Network consumers. International Journal of Marketing Studies, 9(1), 119-128. doi: https://doi.org/10.5539/ijms. v9n1p119 
Cruz, I., y Galán, J. (2019). Determinantes del comportamiento del consumidor en México. Acta Universitaria, 29(1), 1-17. doi: https://doi.org/10.15174/ au.2019.2076

Deaton, A. (1995). Data and econometric tools for development analysis. En J. Behrman y T. Srinivasan (eds.), Handbook of Development Economics (pp. 1785-1882). Amsterdam: Elsevier Science.

Dosi, G. (1988). Sources, procedures, and microeconomic effects of innovation, Journal of economic literature, 26(3), 1120-1171.

Garret, D., y Taumanoff, P. (2010). Are consumers disadvantaged or vulnerable? An examination of consumer complaints to the Better Business Bureau. The Journal of Consumer Affairs, 44(1), 3-23. doi: https://doi.org/10.1111/ j.1745-6606.2010.01155.x

Hess, R., Ganesan, S., y Klein, N. (2003). Service failure and recovery: the impact of relationship factors on customer satisfaction. Journal of the Academy of Marketing Science, 31(2), 127-145. doi: https://doi. org /10.1177/0092070302250898

Hirschman, A. (1970). Exit, voice and loyalty: responses to decline in firm's organizations and states. Cambridge: Harvard University Press.

Hogarth, J., English, M., y Sharma, M. (2001). Consumer complaints and third parties: determinants of consumer satisfaction with complaint resolution efforts. Journal of Consumer Satisfaction, Dissatisfaction, and Complaining Behavior (14), 74-87.

Hsiao, C. (2003). Analysis of Panel Data. Cambridge: Cambridge University Press.

Instituto Nacional de Estadística y Geografía (INEGI). (2019). Encuesta Nacional de Ocupación y Empleo 2019. Recuperado de https://www.inegi.org.mx/ programas/enoe/15ymas/

Jin, L. (2010). Determinants of customers complaint intention: empirical study in the context of China's retail industry. Nankai Business Review International, 1(1), 87-99. doi: https://doi.org/10.1108/20408741011032872

Johnston, R. (1995). Determinants of service quality: satisfiers and dissatisfiers. International Journal of Service Industry Management, 6(5), 53-71. doi: https: / / doi.org/10.1108/09564239510101536

Kim, C., Kim, S., Im, S., y Shin, C. (2003). The effect of attitude and perception on consumer complaint intentions. Journal of Consumer Marketing, 20(4), 352-371. doi: https://doi.org/10.1108/07363760310483702

Kincl, T., y Štrach, P. (2018). Gender differences in online customer satisfaction: the asymmetric and nonlinear effect. Services Marketing Quarterly, 39(3), 157-174, doi: https:// doi.org/10.1080/15332969.2018.1471954

Kolodinsky, J. (1993). Complaints, redress, and subsequent purchases of medical services by dissatisfied consumers. Journal of Consumer Policy, 16(2), 193-214. doi: https: / / doi.org/10.1007/BF01418376

Kolodinsky, J., Murphy, M., Baehr, A., y Lesser, S. (2005). Time price differentials in the rent to own industry: implications for empowering vulnerable consumers. International Journal of Consumer Studies, 29(2), 119-124. doi: https: / / doi.org/10.1111/j.1470-6431.2004.00422.x 
Kwok, S., Jusoh, A., y Khalifah, Z. (2016). The influence of service quality on satisfaction: does gender really matter? Intangible Capital, 12(2), 444-461. doi: http://dx.doi.org/10.3926/ic.673

Larivet, S., y Brouard, F. (2010). Complaints are a firm's best friend. Journal of Strategic Marketing, 18(7), 537-551. doi: https://doi.or$\mathrm{g} / 10.1080 / 0965254 \mathrm{X} .2010 .529155$

Laroche, M., Saad, G., Cleveland, M. , y Browne, E. (2000). Gender differences in information search strategies for a Christmas gift. Journal of Consumer Marketing, 17(6), 500-514. doi: https://doi.org/10.1108/07363760010349920

Martin, I. (1991). Expert-novice differences in complaint scripts. En R. Holman y M. Solomon (eds.), NA-Advances in Consumer Research (pp. 225-231). Provo: Association for Consumer Research.

Maxham III, J., y Netemeyer, R. (2002). A longitudinal study of complaining customers' evaluations of multiple service failures and recovery efforts. Journal of Marketing, 66(3), 57-71. doi: https://doi.org/10.1509/jmkg.66.4.57.18512

Moliner, V., Gil, S., Berenger, C., y Fuentes, B. (2008). Determinantes del comportamiento de queja y su importancia en la segmentación de clientes insatisfechos. Cuadernos de Economía y Dirección de la Empresa, 11(36), 113149. doi: https://doi.org/10.1016/S1138-5758(08)70065-3

Morel, K., Poiesz, T., y Wilke, H. (1997). Motivation, capacity and opportunity to complain: towards a comprehensive model of consumer complaint behavior. Advances in Consumer Research (24), 464-469.

Nerlove, M. (2002). Essays in Panel Data Econometrics. Cambridge: Cambridge University Press.

Prasetyo, A., Hartoyo, H., y Tinaprila, N. (2016). Behavior analysis of consumer complaint. Indonesian Journal of Business and Entrepreneurship, 2(2), 101 111. doi: https://doi.org/10.17358/ijbe.2.2.101

Procuraduría Federal del Consumidor (PROFECO). (2018). Guía de la asesoría y recepción de quejas. Recuperado de https://www.profeco.gob.mx/juridico / Documentos/SSC / Normatividad_Tomol/GUÍA\%20DE\%20LA\%20ASESORÍA\%20Y\%20RECEPCIÓN\%20DE\%20QUEJAS6.pdf

Ringold, D. (2005). Vulnerability in the marketplace: concepts, caveats, and possible solutions. Journal of Macromarketing, 25(4), 202-214. doi: https: / / doi.org/10.1177/0276146705281094

Rosales, R., y Bonilla, J. (2006). Introducción a la econometría. Apuntes de clase núm. 3. CEDE. Santiago de Chile: Facultad de Economía. Universidad de los Andes.

Sánchez, M., y Viana, R. (2017). La relación exportación, investigación y desarrollo tecnológico en América Latina: un análisis de datos de panel dinámico. International Journal of Knowledge Engineering and Managment, 6(14), 96113.

Singh, J. (1988). Consumer complaint intentions and behavior: definitional and taxonomical issues. Journal of Marketing, 52(1), 93-107. doi: https://doi. org/10.2307/1251688

Singh, J. (1989). Determinants of consumers' decisions to seek third party redress: an empirical study of dissatisfied patients. Journal of Consumer 
Affairs, 23(2), 329-63. doi: https://doi.org/10.1111/j.1745-6606.1989. tb00251.x

Singh, J. (1990). Voice, exit, and negative word-of-mouth behaviors: an investigation across three service categories. Journal of the Academy of Marketing Science (18), 1-15. doi: https://doi.org/10.1007/BF02729758

Singh, A., y Vigneswara, I. (2019). Predicting consumer's complaint behavior in telecom services: an empirical study of India, Sri Lanka and Bangladesh. En S. Satapathy y A. Joshi (eds.) Information and Communication Technology for Intelligent Systems. Smart Innovations, Systems and Tecnologies (pp. 465-472), Singapur: Springer.

Singh, J., y Wilkers, R. (1996). When consumers complain: a path analysis of the key antecedents of consumer complaint response estimates. Journal of the Academy of Marketing Science, 24(4), 350-365. doi: https://doi. org/10.1177/0092070396244006

Smith, N., y Cooper-Martin, E. (1997). Ethics and target marketing: the role of product harm and consumer vulnerability. Journal of Marketing, 61(3), 1-20.

Stein, K. (1980). Explaining ghetto consumer behavior: hypotheses from urban sociology. Journal of Consumer Affairs, 14(1), 232-242.

Stephens, N., y Gwinner, K. (1998). Why don't some people complain? A cognitive-emotive process model of consumer complaint behavior. Journal of the Academy of Marketing Science, 26(3), 172-189.

Ursic, M. (1985). A model of the consumer decision to seek legal redress. The Journal of Consumer Affairs, 19(1), 20-36.

Volkov, M., Harker, D., y Harker, M. (2005). Who's complaining? Using MOSAIC to identify the profile of complainants. Marketing Intelligence \& Planning, 23(3), 296-312. doi: https://doi.org/10.1108/02634500510597328

Wahyuni, N., Wardana, I., Yasa, N., y Sukaatmadja, P. (2020). The effect of culture dimension in digitalization era on the complaint behavior in hotel industry. International journal of Data and Network Science, 4(4), 347-356.

Zeelenberg, M., y Pieters, R. (2004). Beyond valence in customer dissatisfaction: A review and new findings on behavioral responses to regret and disappointment in failed services. Journal of Business Research, 57, 445-455. doi: https: / / doi.org/10.1016/S0148-2963(02)00278-3

Zeileis, A. (2004). Econometric computing with HC and HAC covariance matrix estimators. Journal of Statistical Software, 11(10), 1-17. doi: http://dx.doi. org/10.18637/jss.v011.i10 